\title{
INVARIANT SUBSPACES AND SPECTRAL CONDITIONS ON OPERATOR SEMIGROUPS
}

\author{
HEYDAR RADJAVI \\ Department of Mathematics, Dalhousie University \\ Halifax, Nova Scotia, Canada B3H $3 J 5$ \\ E-mail: radjavi@cs.dal.ca
}

0. Introduction. Let $\mathcal{H}$ be a complex Hilbert space of finite or infinite dimension, and let $\mathcal{E}$ be a collection of bounded linear operators on $\mathcal{H}$. We say $\mathcal{E}$ is reducible if there exists a subspace of $\mathcal{H}$, closed by definition and different from the trivial subspaces $\{0\}$ and $\mathcal{H}$ which is invariant under every member of $\mathcal{E}$. We call $\mathcal{E}$ triangularizable if the set of invariant subspaces under $\mathcal{E}$ contains a maximal subspace chain. These questions have been studied extensively and the central problems in the infinite-dimensional case, i.e., the invariant subspace problem and the transitive algebra problem are still unsolved for arbitrary operators on $\mathcal{H}[20,21]$.

We are interested in the effect of certain spectral conditions on reducibility and triangularizability of a collection $\mathcal{E}$. If $f$ is any function, defined at least on all products of members of $\mathcal{E}$, we say that $f$ is permutable if

$$
f\left(A_{1} \ldots A_{k}\right)=f\left(A_{\tau(1)} \ldots A_{\tau(k)}\right)
$$

for every integer $k$, every permutation $\tau$, and all $A_{1}, \ldots, A_{k}$ in $\mathcal{E}$. Special cases of interest for us are when $f$ is the trace, the spectral radius, or the spectrum itself. Permutability of each of these three functions is easily seen to be necessary for triangularizability of a collection of operators at least on a finite-dimensional $\mathcal{H}$.

Since $\mathcal{E}$ will be a multiplicative semigroup in many of our considerations, let us observe that in this case the definition of permutability simplifies for the three functions just named. For example, it follows from the identity $\operatorname{tr}(A B)=\operatorname{tr}(B A)$ and the decomposability of permutations into adjacent transpositions that trace is permutable on a semigroup $\mathcal{E}$ of trace-class operators if and only if $\operatorname{tr}(A B C)=\operatorname{tr}(C B A)$ for all $A, B, C$ in $\mathcal{E}$. The same proof works for the spectral radius since $r(A B)=r(B A)$ for all operators $A$ and $B$. Although the spectrum does not satisfy $\sigma(A B)=\sigma(B A)$ in infinite dimensions,

1991 Mathematics Subject Classification: 47A15, 47D03, 20M20, 15A30.

The paper is in final form and no version of it will be published elsewhere. 
it is still true that it is permutable on a semigroup if and only if $\sigma(A B C)=\sigma(C B A)$ for all $A, B, C$. To verify this, one must only note that if $A$ and $B$ are in the semigroup and if $A B$ is invertible, then the relations

$$
\sigma\left((A B)^{2}\right)=\sigma(A B \cdot A \cdot B)=\sigma(B \cdot A \cdot A B)=\sigma(B A \cdot A B)
$$

imply that $(B A)(A B)$ is invertible and so is $B A$. This means that in a semigroup satisfying the condition $\sigma(A B C)=\sigma(C B A)$, the identity $\sigma(A B)=\sigma(B A)$ holds, so that, just as in the cases of trace and spectral radius, this condition implies permutability of the spectrum.

A second kind of spectral condition we shall consider is submultiplicativity. We say the spectral radius is submultiplicative on $\mathcal{E}$ if

$$
r(A B) \leq r(A) r(B)
$$

for all $A$ and $B$ in $\mathcal{E}$. The spectrum itself is said to be submultiplicative on $\mathcal{E}$ if the condition

$$
\sigma(A B) \subseteq \sigma(A) \sigma(B)
$$

holds for all $A$ and $B$ in $\mathcal{E}$. By $\sigma(A) \sigma(B)$ we mean, as usual, the set

$$
\{\alpha \beta: \alpha \in \sigma(A), \beta \in \sigma(B)\} .
$$

Like permutability, these submultiplicativity conditions are also obviously necessary for triangularization in finite dimensions.

In what follows, by a semigroup we shall just mean a collection closed under multiplication.

1. Permutability in finite dimensions. Perhaps one of the most well-known triangularizability results is Levitzki's result [11]: a semigroup of nilpotent operators (on a finite-dimensional space) is triangularizable. If "nilpotent" is replaced by "unipotent", one obtains Kolchin's Theorem [8]. (An operator $A$ is called unipotent if $\sigma(A)=\{1\}$.) In both of these results trace is constant on the semigroup (and therefore permutable). Kaplansky [8] unified the Levitzki and Kolchin theorems by proving the following result.

THEOREM 1. A semigroup $\mathcal{S}$ with constant trace is triangularizable. Furthermore, constancy of the trace implies that $\sigma(A) \subseteq\{0,1\}$ for every $A$ in $\mathcal{S}$, where 1 has a fixed algebraic multiplicity $k$.

There are many other sufficient conditions for triangularizability of collections, e.g., the well-known elementary exercise in linear algebra that a commutative collection is triangularizable. It turns out that permutability of trace, which is obviously necessary, is also sufficient for triangularizability [16].

TheOREM 2. A set of operators is triangularizable if and only if trace is permutable on it.

The most elementary proof of Theorem 2 is perhaps given in [17] which proves and uses the following extension of Engel's Theorem: if $\mathcal{E}$ is a set of operators closed under the Lie bracket $[A, B]=A B-B A$, then $\mathcal{E}$ is triangularizable if and only if $A B-B A$ is nilpotent for every $A$ and $B$ in $\mathcal{E}$. Given this result, here is how one deduces Theorem 2 . 
Let trace be permutable on a semigroup $\mathcal{S}$. It is easily verified that it is permutable on the linear span of $\mathcal{S}$, i.e., the algebra $\mathcal{A}$ generated by $\mathcal{S}$. Now we will be done if we show that the set

$$
\{A B-B A: A, B \in \mathcal{A}\}
$$

consists of nilpotents, This can be accomplished by verifying that the positive powers of $A B-B A$ all have trace zero. But the permutability of trace implies that, for every $k$,

$$
\operatorname{tr}\left((A B-B A)^{k}\right)=\sum_{m=0}^{k}(-1)^{m}\left(\begin{array}{c}
k \\
m
\end{array}\right) \operatorname{tr}\left(A^{k} B^{k}\right)=0 .
$$

A nonobvious corollary of Theorem 2 is the following result on bands. A band means a semigroup of idempotents.

COROLlary 1. A band of operators is triangularizable.

Proof. Denote the rank of $A$ by $\varrho(A)$ and observe that $\varrho(A)=\operatorname{tr}(A)$ for any idempotent $A$. Let $\mathcal{S}$ be a band. If $A$ and $B$ are in $\mathcal{S}$, then

$$
\varrho(B A B)=\operatorname{tr}(B A B)=\operatorname{tr}\left(B^{2} A\right)=\operatorname{tr}(B A)=\varrho(B A) .
$$

But the range of $B A B$ is always contained in that of $B A$, so the equation above shows that these ranges coincide. Thus $C B A B$ and $C B A$ have the same range for every $C$ in $\mathcal{S}$, yielding $\varrho(C B A B)=\varrho(C B A)$. Interchanging $A$ and $C$ we also obtain $\varrho(A B C B)=$ $\varrho(A B C)$. These two equations together with

$$
\varrho(A B C B)=\operatorname{tr}(A B C B)=\operatorname{tr}(C B A B)=\varrho(C B A B)
$$

yield the desired relation $\operatorname{tr}(A B C)=\operatorname{tr}(C B A)$ for all $A, B$, and $C$ in the band. Thus $\mathcal{S}$ is triangularizable by Theorem 2 .

We note here that this corollary is true for a band over an arbitrary field, as are some other results in this paper, but we confine ourselves to the complex field.

Classifying bands of operators is an interesting but seemingly, and perhaps surprisingly, nontrivial task. A start is made in [3].

There are other, easy, corollaries of Theorem 2.

COROLlary 2. If $\mathcal{G}$ is a group of operators, then $\mathcal{G}$ is triangularizable if and only if trace is constant on each coset of $\mathcal{G}$ relative to the commutator subgroup.

COROllary 3. A self-adjoint set of operators is commutative if and only if trace is permutable on it.

If an arbitrary linear functional is considered in place of trace, one cannot always get triangularizability. Reducibility is all that could be hoped for.

Proposition 1. Let $\phi$ be a nonzero linear functional on the algebra of all operators. If $\phi$ is permutable on a set $\mathcal{E}$ of operators, then $\mathcal{E}$ is reducible.

The proof is easy; it uses the fact that if a nonzero ideal in an algebra $\mathcal{A}$ is reducible, so is $\mathcal{A}$ itself. Now the permutability of $\phi$ on $\mathcal{E}$ implies the same for the algebra $\mathcal{A}$ generated by $\mathcal{E}$. For commutative $\mathcal{A}$ there is nothing to prove. Otherwise, pick $A B-B A \neq 0$ in $\mathcal{A}$ and let $\mathcal{J}$ be the ideal generated by $A B-B A$. The permutability condition implies 
that $\phi$ is zero on $\mathcal{J}$. This shows that $\mathcal{J}$ is reducible by Burnside's Theorem: every proper subalgebra of operators on $\mathbb{C}^{n}$ is reducible [4].

An obvious corollary of Proposition 1 is that if a nonzero functional $\phi$ is constant on a semigroup or, more generally, if it is multiplicative, then the semigroup is reducible. This simple proposition and its corollaries are used in the proofs of some results to follow.

We next consider the permutability of the spectrum. (The weaker condition of permutability of the spectral radius will be discussed in connection with submultiplicativity.) This turns out to be quite a strong condition for groups, but not so strong for semigroups [10].

THEOREM 3. Every group of operators with permutable spectrum is triangularizable. Every semigroup satisfying this condition (on a space of dimension greater than 1) is reducible.

One ingredient in the proof of the group case [10] is that the commutator subgroup consists of unipotent operators by the permutability hypothesis, and is thus triangularizable by Kolchin's result.

It is easy to give examples of nontriangularizable semigroups with permutable spectrum. For instance, let $n \geq 2$ and let $\mathcal{E}$ be the collection of all "basic" matrices, that is, those with one entry 1 and all other entries zero. Let $\mathcal{S}$ be the semigroup of all $(n+1) \times(n+1)$ matrices of the form

$$
\left\{\left(\begin{array}{cc}
1 & X \\
0 & Y
\end{array}\right): X \text { arbitrary, } Y \in \mathcal{E} \cup\{0\}\right\} .
$$

Then $\sigma(S)=\{0,1\}$ for every $S$ in $\mathcal{S}$, i.e., spectrum is even constant on $\mathcal{S}$, and yet $\mathcal{S}$ has only one nontrivial invariant subspace.

We should note here that we are speaking of the spectrum of an operator as a set, with no regard for algebraic multiplicities. If one assumes the stronger condition that, for every $A, B, C$ in a semigroup, $A B C$ and $C B A$ have the same spectrum with the same respective multiplicities, then the permutability of trace and thus triangularizability follows for the semigroup.

2. Submultiplicativity in finite dimensions. The group of unitary operators is the best example to show that the submultiplicativity of spectral radius by itself cannot yield reducibility. (In this example $r$ is multiplicative, even constant, and thus also permutable.) However, in the presence of additional conditions [18, 19] affirmative results can be obtained. Here is a sample result [19].

THEOREM 4. Let $\mathcal{S}$ be a semigroup of operators represented (simultaneously) by matrices with nonnegative entries. If $r(A B) \leq r(A) r(B)$ for all $A$ and $B$ in $\mathcal{S}$, then $\mathcal{S}$ is reducible.

Let us mention certain facts, of some interest in themselves, that are used in the proof of this and other similar results.

(1) If $\mathcal{S}$ is a semigroup of $n \times n$ matrices with nonnegative entries and $\sigma(S)$ is contained in the unit circle for every $S$ in $\mathcal{S}$, then $\mathcal{S}$ is reducible (if $n>1$ ) [19]. 
More generally, let $\mathcal{S}$ be a semigroup of matrices with nonnegative entries and let $P$ be an idempotent of rank $\geq 2$ in $\mathcal{S}$. Assume that for every $S \in \mathcal{S}$, the spectrum of the operator $P S P$, when restricted to the range of $P$, is entirely on the unit circle. Then $P \mathcal{S} P$ has a nonzero invariant subspace properly contained in the range of $P$.

(2) No irreducible semigroup $\mathcal{S}$ with submultiplicative spectral radius can contain nonzero nilpotents. Assume otherwise. Then the nilpotents in $\mathcal{S}$ would form a semigroup ideal $\mathcal{J}$. (This means that for every $J$ in $\mathcal{J}$ and $S \in \mathcal{S}$ both $S J$ and $J S$ belong to $\mathcal{J}$.) Now $\mathcal{J}$ is reducible by Levitzki's Theorem and, just as in the case of algebras, it can be verified easily that the reducibility of a nonzero ideal implies that of the semigroup itself.

(3) An irreducible semigroup $\mathcal{S}$ with submultiplicative $r$ cannot contain divisors of zero. For if $A B=0$ with nonzero $A$ and $B$ in $\mathcal{S}$, then $(B S A)^{2}=0$ for every member $S$ of $\mathcal{S}$ and thus $B S A=0$ by the preceding paragraph. Therefore, the range of $A$ generates an invariant subspace $\mathcal{M}$ of $\mathcal{S}$ contained in the kernel of $B$. (This subspace is the span of all $S A x$, with $x$ in the space and $S$ in $\mathcal{S}$.)

(4) If $A$ is any operator with $r(A)=1$. Then the closure of the set

$$
\left\{\frac{1}{m} A^{n}: m \text { and } n \text { positive integers }\right\}
$$

contains a nonzero operator which is either nilpotent or idempotent. To see this we can express $A$ as a direct sum $A_{1} \oplus A_{2}$, where $\sigma\left(A_{1}\right)$ and $\sigma\left(A_{2}\right)$ are, respectively, on the unit circle and in the open unit disc. Since the powers of $A_{2}$ tend to zero, we can assume $A=A_{1}$ for our purposes. Now $A$ is similar to an operator $U+N$ with $U$ unitary and $N$ a nilpotent operator commuting with $U$. There is no harm in assuming $A=U+N$. If $N=0$, observe that some subsequence of $\left\langle U^{n}\right\rangle$ tends to the identity operator, and we are done. If $N^{k} \neq 0$ and $N^{k+1}=0$ for some positive integer $k$, then consider the expansion

$$
(U+N)^{n}=U^{n}+\left(\begin{array}{l}
n \\
1
\end{array}\right) U^{n-1} N+\ldots+\left(\begin{array}{l}
n \\
k
\end{array}\right) U^{n-k} N^{k}
$$

and note that some subsequence of $\left\langle U^{n-k} N^{k}\right\rangle$ approaches $N^{k}$. Thus the corresponding subsequence of $A^{n} /\left(\begin{array}{l}n \\ k\end{array}\right)$ has limit $N^{k}$.

Let us use these facts to prove a special case of the preceding theorem, where we make the stronger hypothesis of multiplicativity for $r$. First observe that there is no loss of generality in assuming that $\mathcal{S}$ is closed (in norm), by continuity of the spectral radius. There is also no harm in assuming that $\mathcal{S}$ is closed under multiplication by positive scalars. In view of (3) above, we can assume $\mathcal{S}$ has no divisors of zero, so that it can be replaced by $\mathcal{S} \backslash\{0\}$ if $0 \in \mathcal{S}$. Also assume $r(S) \neq 0$ for all $S$, by (2).

Pick a member $A$ of $\mathcal{S}$ with minimal rank and $r(A)=1$. By (2) and (4), we can assume that $\mathcal{S}$ contains an idempotent $P$ as a limit of powers of $A$.

Replacing $\mathcal{S}$ by $\{S / r(S): S \in \mathcal{S}\}$, we can now assume that every member has spectral radius 1 . So the subsemigroup $P \mathcal{S} P$, when restricted to the range of $P$, consists of operators with spectrum on the unit circle, by the minimality of the rank $k$ of $P$. Thus, if $k>1$, then some nontrivial subspace of the range of $P$ is invariant under $P \mathcal{S} P$ by (1) above. This implies the existence of nonzero vectors $x=P x$ and $y=P y$ such that the 
inner product $(P S P x, y)$ is zero for all $S$, so that $\mathcal{S}$ has an invariant subspace perpendicular to $y$. If $k=1$, then it is easily seen that $P S P=P$ for all $S$. This yields a functional $(P S P x, x)=1$ on the semigroup, which implies reducibility by Proposition 1.

In light of the following result from [13], Theorem 4 reduces to the special case we have just proved.

THEOREM 5. If the spectral radius is submultiplicative on an irreducible semigroup, then it is multiplicative.

Obvious examples of irreducible semigroups with multiplicative $r$ are unitary groups, but there are other, more interesting examples. These semigroups are studied in [15].

The theorem above is itself a special case of the following theorem [13] on arbitrary (not necessarily irreducible) semigroups.

THEOREM 6. The spectral radius is submultiplicative on a semigroup if and only if it is permutable.

More on this later.

A condition much stronger that the submultiplicativity of the spectral radius is that of spectrum itself: for every $S$ and $T$ in a semigroup

$$
\sigma(S T) \subseteq \sigma(S) \sigma(T) .
$$

Like permutability, this condition is clearly necessary for triangularizability. However, there are finite groups of $n \times n$ matrices for every odd $n$ that satisfy this condition but are irreducible [10]. To form such a group for an odd prime $p$, let $\Omega$ be the set of complex $p$ th roots of unity and let $\mathcal{D}$ denote the group of all diagonal matrices $\operatorname{diag}\left(w_{1}, \ldots, w_{p}\right)$ with determinant 1 and $w_{i} \in \Omega, i=1, \ldots, p$. Let

$$
U=\left(\begin{array}{cccccc}
0 & 0 & 0 & \ldots & 0 & 1 \\
1 & 0 & 0 & \ldots & 0 & 0 \\
0 & 1 & 0 & \ldots & 0 & 0 \\
\ldots & \ldots & \ldots & \ldots & \ldots \\
0 & 0 & 0 & \ldots & 0 & 0 \\
0 & 0 & 0 & \ldots & 1 & 0
\end{array}\right)
$$

and let $\mathcal{G}_{p}$ be the group generated by $\mathcal{D}$ and $U$. It is not hard to verify that $\sigma(G)=\Omega$ for all nondiagonal $G$. It can also be verified, by distinguishing a few cases, that $\sigma$ is submultiplicative on $\mathcal{G}_{p}$. That $\mathcal{G}$ is irreducible can also be checked easily.

For a composite odd $n=p_{1} \ldots p_{k}$, with (not necessarily distinct) primes $p_{i}$, let $\mathcal{G}$ be the tensor product of the $\mathcal{G}_{p_{i}}$ and observe that $\sigma(A \otimes B)=\sigma(A) \sigma(B)$ for any $A$ and $B$.

A semigroup with submultiplicative spectrum is reducible if it has a nonzero singular member.

3. The case of compact operators. Many of the results we have listed have extensions to the appropriate sets of compact operators in the infinite-dimensional case. For example, Theorem 2 holds when it makes sense, that is, for operators in the trace class $\mathcal{C}_{1}$ [16]. So do Corollaries 1 and 3. Extensions of results on trace permutability are treated in [6]. Proposition 1 is true for compact operators; the functional $\phi$ should of course be 
assumed continuous and not zero on the algebra of all compact operators. Or, one could restrict the operators to the class $\mathcal{C}_{1}$, and require $\phi$ to be a nonzero functional on $\mathcal{C}_{1}$, continuous in trace norm.

The relevant part of Theorem 3, considered for general compact operators, poses a problem. The following special case of it remains unresolved [14, 23].

QUESTION 1. Is a semigroup of compact quasinilpotent operators reducible?

If we confine ourselves to the class $\mathcal{C}_{1}$, or to any larger Schatten class $\mathcal{C}_{p}$, the answer is affirmative; in fact, it suffices to assume the presence of one nonzero member in the semigroup that belongs to some Schatten class [14]. Of course, if the semigroup is entirely contained in $\mathcal{C}_{1}$, it is even triangularizable by Theorem 2 .

The well-known Lomonosov Theorem [12] implies that if the semigroup in Question 1 is an algebra, then it is triangularizable. This result and Ringrose's work on diagonal coefficients for compact operators [22] are important ingredients in proofs of reducibility and triangularizability for collections of compact operators. For example, they yield the following theorem [9], which also holds for operators on Banach spaces.

TheOREM 7. An algebra $\mathcal{A}$ of compact operators is triangularizable if and only if $A B-B A$ is quasinilpotent for all $A$ and $B$ in $\mathcal{A}$.

This theorem can be used to give a simple proof of Theorem 2 applied to trace class operators: Let $\mathcal{S}$ be any semigroup that has permutable trace. Then so does the algebra $\mathcal{A}$ generated by $\mathcal{S}$. If $A$ and $B$ are in $\mathcal{A}$, then $\operatorname{tr}\left((A B-B A)^{k}\right)=0$ for all $k$, as in the proof of Theorem 2 in the finite-dimensional case. This shows that $\sigma(A B-B A)=0$, and thus $\mathcal{A}$ is triangularizable. The converse also follows from Ringrose's results.

It is possible to avoid Question 1, as in the following result from [10].

THEOREM 8. Let $\mathcal{S}$ be a semigroup of operators with permutable spectrum. If $\mathcal{S}$ contains a nonquasinilpotent compact operator, then $\mathcal{S}$ is reducible.

Note that $\mathcal{S}$ itself is not assumed to be contained in the set of compact operators. This is because, as discussed earlier, one can always consider the semigroup ideal of compact operators in $\mathcal{S}$. If the ideal is reducible, so is the semigroup.

We turn now to submultiplicativity conditions. Theorem 4 holds verbatim for semigroups of compact operators. More generally, it holds whenever $\mathcal{S}$ contains some nonzero compact operator [10]. The proof is not essentially different from that outlined in Section 2 ; this is why we dwelt on it so long there. Extensions of these results using positivity are treated in $[2,26]$.

Theorems 5 and 6 are valid for semigroups of compact operators [13]. An obvious corollary is that, as in the finite-dimensional case, if the spectral radius is submultiplicative on a semigroup of compact operators but not multiplicative, then there is a nontrivial invariant subspace.

The stronger condition of submultiplicativity for spectrum itself is a little different in infinite dimensions, because we cannot have groups of compact operators. On the other hand, we must avoid the unsettled Question 1. 
ThEOREM 9. Let $\mathcal{S}$ be any semigroup of operators on infinite-dimensional Hilbert space containing a nonquasinilpotent compact operator. If $\sigma(S T) \subseteq \sigma(S) \sigma(T)$ for all $S$ and $T$ in $\mathcal{S}$, then $\mathcal{S}$ is reducible.

This theorem and other results using submultiplicativity conditions are proved in [10].

For other related results see Shul'man's paper [24].

4. General operators in infinite dimensions. Many assertions made so far fail to hold in infinite dimensions, some spectacularly. For example, consider a semigroup $\mathcal{S}$ of operators with $\sigma(S)=\{0\}$ for every $S \in \mathcal{S}$. In finite dimensions and in the trace-class case, such an $\mathcal{S}$ is triangularizable. In the general compact case, we are in the unsettled situation of Question 1. As soon as we drop the compactness condition, however, there is a "very irreducible" semigroup $\mathcal{S}$ of "very nilpotent" operators. The first "very" means that $\mathcal{S}$ is not just irreducible, but (what is apparently a stronger state of affairs) weak-operator dense in the algebra of all operators [6,7]. The second "very" refers to the fact that every member $S$ of $\mathcal{S}$ satisfies $S^{2}=0$. This example is given in [6] and is constructed as follows.

Fix an orthonormal basis for $\mathcal{H}$. Every operator will be represented by its matrix relative to this basis. For a given square matrix $M$ of finite size, we write $M \oplus \ldots$ to indicate the inflation of $M$, i.e., the direct sum of infinitely many summands all equal to $M$. For every positive integer $n$, let $\mathcal{M}(n)$ denote the set of complex $n \times n$ matrices and let

$$
\mathcal{S}_{k}=\left\{\left(\begin{array}{cc}
A & A \\
-A & -A
\end{array}\right) \oplus \ldots: A \in \mathcal{M}\left(2^{k-1}\right)\right\}
$$

for $k=1,2, \ldots$ It is easily verified that $\mathcal{S}_{k}$ is a semigroup of operators of square zero. Furthermore, if $S \in \mathcal{S}_{k}$ and $T \in \mathcal{S}_{m}$ and $k<m$, then $S T$ and $T S$ both belong to $\mathcal{S}_{m}$. Thus $\mathcal{S}=\bigcup_{k=1}^{\infty} \mathcal{S}_{k}$ is a semigroup of nilpotents of index two. It is not difficult to show that the closure of $\mathcal{S}$ in the weak operator topology is $\mathcal{B}(\mathcal{H})$.

One can go further with this example and form a dense algebra of nilpotent operators. Just let $\mathcal{A}$ be the linear span of $\mathcal{S}$. Since $\mathcal{S}$ is closed under multiplication by scalars, every member of $\mathcal{A}$ is simply a sum $A=S_{1}+\ldots+S_{k}$. It can be shown by induction that $A^{2^{k}}=0$. Observe that there are nilpotents of arbitrarily high index in $\mathcal{A}$. In fact an algebra of nilpotents of bounded index is triangularizable [7].

What about a band in general? We have seen that a band of compact operators is triangularizable. (A compact idempotent is necessarily of finite rank. Thus the proof given for Corollary 1 applies, showing that trace is permutable.) Of course, to get just one invariant subspace, we only need one nonzero finite-rank idempotent: An arbitrary band with at least a nonzero finite-rank member is reducible. The general question is unsettled.

QUESTION 2. Is a band of operators reducible?

There are some results and more questions on permutability and submultiplicativity. We shall confine ourselves to the spectral radius here. An interesting result of Aupetit and Zemánek [1, 25] gives several equivalent conditions involving the spectral radius. Note 
that this theorem is true for a general Banach algebra, not just a norm-closed algebra of operators.

Theorem 10. For a Banach algebra $\mathcal{A}$ the following conditions are mutually equivalent:

(1) $\mathcal{A} / \operatorname{Rad} \mathcal{A}$ is abelian.

(2) $r(a b) \leq r(a) r(b)$ for all $a, b$ in $\mathcal{A}$.

(3) $r(a+b) \leq r(a)+r(b)$ for all $a, b$ in $\mathcal{A}$.

It is interesting that these conditions are also equivalent to permutability [10]. If $\mathcal{S}$ is any multiplicative semigroup in a Banach algebra $\mathcal{A}$, then permutability of $r$ on $\mathcal{S}$ implies its submultiplicativity. This is easy to see [10]: Let $a$ and $b$ be arbitrary members of $\mathcal{S}$. For positive integer $n$, the permutability of $r$ implies that

$$
r\left[(a b)^{n}\right]=r\left(a^{n} b^{n}\right) \leq\left\|a^{n} b^{n}\right\| \leq\left\|a^{n}\right\| \cdot\left\|b^{b}\right\| .
$$

Hence $[r(a b)]^{n} \leq\left\|a^{n}\right\| \cdot\left\|b^{n}\right\|$. Taking roots of both sides and letting $n$ approach infinity, we obtain $r(a b) \leq r(a) r(b)$

The converse is the content of the following open question.

QUESTION 3. Let $\mathcal{S}$ be a semigroup in a Banach algebra. Does the submultiplicativity of $r$ on $\mathcal{S}$ imply its permutability?

A special case of the question is, of course, the one in which we restrict ourselves to operators. Let us include here the very short proof [10] that the answer is yes when $\mathcal{S}$ is a Banach algebra: Let $a, b, c$ be arbitrary members of $\mathcal{A}$, where $\mathcal{A}$ satisfies Condition (2), and thus all conditions of the Aupetit-Zemánek theorem above. Then, by Condition (3),

$$
r(a b c) \leq r(a b c-b a c)+r(b a c)=r[(a b-b a) c]+r(b a c) .
$$

Now (1) implies that $(a b-b a) c$ is quasinilpotent and thus $r(a b c) \leq r(b a c)$. Interchanging $a$ and $b$ we get $r(a b c)=r(b a c)$.

If $\mathcal{S}$ is merely a semigroup, the question is answered affirmatively in some cases. The case of compact operators has already been mentioned. (This includes the affirmative answer in finite dimensions.) Another case is where $\mathcal{S}$ is contained in the set of normal operators. In this case $r$, being equal to the norm, is automatically submultiplicative. Its permutability is shown in [13], where some other cases are also treated.

\section{References}

[1] B. Aupetit, Propriétés spectrales des algèbres de Banach, Lecture Notes in Math. 735, Springer, Berlin, 1979.

[2] M. D. Choi, E. Nordgren, H. Radjavi, P. Rosenthal and Y. Zhong, Triangularizing semigroups of quasinilpotent operators with nonnegative entries, Indiana Univ. Math. J. 42 (1993), 15-25.

[3] P. Fillmore, G. MacDonald, M. Radjabalipour, and H. Radjavi, Towards a classification of maximal unicellular bands, Semigroup Forum 49 (1994), 195-215.

[4] N. Jacobson, Lectures in Abstract Algebra II: Linear Algebra, Van Nostrand, Princeton, 1953. 
[5] D. Hadwin, Radjavi's trace condition for triangularizability, J. Algebra 109 (1987), 184192.

[6] D. Hadwin, E. Nordgren, M. Radjabalipour, H. Radjavi and P. Rosenthal, A nil algebra of bounded operators on Hilbert space with semisimple norm closure, Integral Equations Operator Theory 9 (1986), 739-743.

[7] -, On simultaneous triangularization of collections of operators, Houston J. Math. 17 (1991), 581-602.

[8] I. Kaplansky, The Engel-Kolchin theorem revisited, in: Contributions to Algebra, H. Bass, P. J. Cassidy, and J. Kovacik (eds.), Academic Press, New York, 1977, 233237.

[9] A. Katavolos and H. Radjavi, Simultaneous triangularization of operators on a Banach space, J. London Math. Soc. (2) 41 (1990), 547-554.

[10] L. Lambrou, W. Longstaff and H. Radjavi, Spectral conditions and reducibility of operator semigroups, Indiana Univ. Math. J. 41 (1992), 449-464.

[11] J. Levitzki, Über nilpotente Unterringe, Math. Ann. 105 (1931), 620-627.

[12] V. I. Lomonosov, Invariant subspaces for the family of operators commuting with compact operators, Funktsional. Anal. i Prilozhen. 7 (3) (1973), 55-56 (in Russian).

[13] W. Longstaff and H. Radjavi, On permutability and submultiplicativity of spectral radius, Canad. J. Math. 47 (1995), 1007-1022.

[14] E. Nordgren, H. Radjavi and P. Rosenthal, Triangularizing semigroups of compact operators, Indiana Univ. Math. J. 33 (1984), 271-275.

[15] M. Omladič and H. Radjavi, Irreducible semigroups with multiplicative spectral radius, Linear Algebra Appl. 251 (1997), 59-72.

[16] H. Radjavi, A trace condition equivalent to simultaneous triangularizability, Canad. J. Math. 38 (1986), 376-386.

[17] —, The Engel-Jacobson theorem revisited, J. Algebra 111 (1987), 427-430.

[18] - On the reduction and triangularization of semigroups of operators, J. Operator Theory 13 (1985), 63-71.

[19] - On reducibility of semigroups of compact operators, Indiana Univ. Math. J. 39 (1990), 499-515.

[20] H. Radjavi and P. Rosenthal, Invariant Subspaces, Springer, Berlin, 1973.

[21] —, - , The invariant subspace problem, Math. Intelligencer 4 (1982), 33-37.

[22] J. Ringrose, Super diagonal forms for compact linear operators, Proc. London Math. Soc. (3) 12 (1962), 367-384.

[23] V. S. Shul'man, On invariant subspaces of Volterra operators, Funktsional. Anal. i Prilozhen. 18 (2) (1984), 85-86 (in Russian).

[24] - Invariant subspaces and spectral mapping theorems, in: Functional Analysis and Operator Theory, Banach Center Publ. 30, Inst. Math., Polish Acad. Sci., Warszawa, 1994, 313-325.

[25] J. Zemánek, Properties of the spectral radius in Banach algebras, in: Spectral Theory, Banach Center Publ. 8, PWN, Warszawa, 1982, 579-595.

[26] Y.Zhong, Functional positivity and invariant subspaces of semigroups of operators, Houston J. Math. 19 (1993), 239-262.

Editorial note: See also R. Drnovšek, An irreducible semigroup of idempotents, Studia Math., to appear. 